\title{
A FRONT-TRACKING ALTERNATIVE TO THE RANDOM CHOICE METHOD
}

\author{
NILS HENRIK RISEBRO
}

(Communicated by Barbara L. Keyfitz)

\begin{abstract}
An alternative to Glimm's proof of the existence of solutions to systems of hyperbolic conservation laws is presented. The proof is based on an idea by Dafermos for the single conservation law and in some respects simplifies Glimm's original argument. The proof is based on construction of approximate solutions of which a subsequence converges. It is shown that the constructed solution satisfies Lax's entropy inequalities. The construction also gives a numerical method for solving such systems.
\end{abstract}

\section{INTRODUCTION}

We study the initial value problem for the general system of hyperbolic conservation laws

$$
u_{t}+f(u)_{x}=0 .
$$

Our analysis is based on Lax's [1] solution of the Riemann problem. We give here an alternative proof of Glimm's fundamental result [2] not based on a random sequence. Since Glimm's paper, there have been few generalizations of his result, but Liu [3] showed that Glimm's proof did not actually depend on the random sequence and that it converged for any equidistributed sequence. Chorin [4] developed Glimm's construction into a numerical method. Using Glimm's construction, Lax [5] showed that the conctructed solution satisfied the entropy inequalities provided the system admitted an additional conservation law. This system of equations models a diverse range of physical phenomena, e.g., traffic flow [6], gas dynamics [7], and multiphase flow in porous media [8].

Our proof is based on ideas from the study of the single conservation law. Dafermos [9] used a piecewise linear continuous approximation to the flux function $f$ to obtain approximate piecewise constant solutions. This idea was further developed into a numerical method by LeVeque [10] and by Holden et al. [11] and was generalized to several space dimensions by Høegh-Krohn and Risebro [12]. The idea of approximating rarefaction waves by piecewise constant states was also investigated by Swartz and Wendroff [13] for the system of gas dynamics.

Received by the editors October 24, 1989 and, in revised form, August 8, 1991.

1991 Mathematics Subject Classification. Primary 35L65, 35D05, 76T05.

The author has been supported by Statoil and the Royal Norwegian Council for Technical and Industrial Research. 
We construct our solutions by starting with an approximation to the solution of the Riemann problem where the rarefaction part of the solution is replaced by an approximating step function. The inital value function is also approximated by a step function, which defines a series of Riemann problems. Each discontinuity in the approximate solution is then tracked until it interacts with other discontinuities. For such interactions we can use some of the estimates in [2] directly, and here we only give the differences from Glimm's proof. Our main result is that if the total variation of the initial data is small, then a weak solution of the initial value problem exists. Without assuming the existence of an additional conservation law, we show that our constructed solution satisfies Lax's entropy inequalities, and therefore is not of what Glimm [2] called "extranous" type. The construction in a natural way defines a numerical method for solving hyperbolic conservation laws. For general background we refer the reader to [14, part 3] and the references therein.

\section{Method AND NOTATION}

We will consider the Cauchy problem

$$
\begin{aligned}
& u_{t}+f(u)_{x}=0 \\
& u(x, 0)=u_{0}(x)
\end{aligned}
$$

where $f: \mathbb{R}^{n} \rightarrow \mathbb{R}^{n}$ is a smooth function. We assume that the system is strictly hyperbolic, that is, the Jacobian $d f$ has real eigenvalues $\lambda_{1}(u), \ldots, \lambda_{n}(u)$ such that $\lambda_{1}(u)<\lambda_{2}(u)<\cdots<\lambda_{n}(u)$. We want to construct a weak solution to $(2.1)$, that is, a function $u: \mathbb{R} \times \mathbb{R}^{+} \rightarrow \mathbb{R}^{n}$

$$
\int_{0}^{\infty} \int_{-\infty}^{\infty}\left(\phi_{t} u+\phi_{x} f(u)\right) d x d t+\int_{-\infty}^{\infty} \phi(x, 0) u_{0}(x) d x=0
$$

for all smooth $\phi$ with compact support in $(x, t)$.

The Riemann problem for $(2.1)$ is the initial value problem where

$$
u_{0}(x)= \begin{cases}u_{l}, & x<0 \\ u_{r}, & x>0\end{cases}
$$

The solution of this Riemann problem consists of three ingredients: shocks, rarefaction waves, and contact discontinuities. For an explanation of these see [14, Chapter 17].

Theorem 1 (Lax). Let $u_{l} \in N \subset \mathbb{R}^{n}$ and suppose (2.1) is strictly hyperbolic and that each characteristic field is either genuinely nonlinear or linearly degenerate in $N$. Then there is a neighbourhood $M \subset N$ such that for $u_{r} \in M$, (2.3) has a solution. This solution consists of at most $n+1$ constant states separated by shocks, rarefaction waves, and contact discontinuities. There is only one such solution in $M$, and discontinuities in this solution will satisfy the Lax inequalities (3.13).

Proof. See [14, Theorem 17.18].

We will construct an approximation to this solution. Through each point $u$ in $M$ we have $n$ one-parameter families of curves $U_{k}(u, \varepsilon), k=1, \ldots, n$. These have continuous derivatives of order two at $\varepsilon=0$ and have the property that if $u_{r}$ is on $U_{k}\left(u_{l}, \varepsilon\right)$ and the $k$ th field is genuinely nonlinear, $u_{r}$ can 
be connected to $u_{l}$ by a rarefaction wave iff $\varepsilon>0$ and by a shock iff $\varepsilon<0$, $U_{k}\left(u_{l}, 0\right)=u_{l}$. We call $|\varepsilon|$ the strength of the wave. If the $k$ th field is linearly degenerate $U_{k}(u, \varepsilon)$ consists of the states that can be connected to $u$ by a contact discontinuity. For a more detailed description of these concepts we again refer to [14].

We will take this correct solution to the Riemann problem and approximate it in the following way: We start with the correct solution to (2.3). Leave each shock or contact discontinuity as it is. Along the rarefaction curves, we fix an initial $\delta>0$, approximate the rarefaction fan by constant states

$$
u_{i}^{(k)}=U_{k}\left(u^{k}, i \delta\right)=U_{k}\left(u_{i-1}^{(k)}, \delta\right)
$$

for $i=1, \ldots, m$, where $m$ is chosen such that $u_{m+2}^{(k)}$ is "past" the next constant state in the solution: $u^{k+1}$. The states $u_{i}^{(k)}$ and $u_{i+1}^{(k)}$ will be separated by a discontinuity moving with speed $\lambda_{k}\left(u_{i+1}^{(k)}\right)$. This approximation corresponds to making a step function approximation of $u(x, t)$ at each fixed $t$. We call our approximation $u_{\delta}(x, t)$. We have that

$$
\lim _{\delta \rightarrow 0} u_{\delta}=u \quad \text { for all } t .
$$

The limit is in $L_{1}^{\text {loc }}(\mathbb{R}, d x)$ for each $t$. Furthermore $u$ will satisfy (2.2), and since supp $\phi$ is confined to $t<T<\infty$, we have that

$$
\int_{0}^{\infty} \int_{-\infty}^{\infty}\left(\phi_{t} u_{\delta}+\phi_{x} f\left(u_{\delta}\right)\right) d x d t+\int_{-\infty}^{\infty} \phi(x, 0) u_{0}(x) d x \rightarrow 0
$$

as $\delta \rightarrow 0$, since, by the bounded convergence theorem, $f\left(u_{\delta}\right) \rightarrow f(u)$ in $L_{1}$. Therefore $u_{\delta}$ is an approximate solution to (2.3), and we will call this a $\delta$ approximation to the solution of (2.3).

Our strategy will now be to construct an approximation to a more general initial value problem, by using this $\delta$-approximation on a series of Riemann problems. Assume that $u_{0}(x)$ is in $L_{1}^{\text {loc }} \cap B . V$., then we define the sequence $\left\{x_{i}\right\}_{1}^{M}$ by

$$
\begin{gathered}
x_{1}=\inf \left\{x:\left|u_{0}(x)-\lim _{x \rightarrow-\infty} u_{0}(x)\right|>\delta\right\} \\
x_{n+1}=\inf \left\{x: x>x_{n} \text { and }\left|u_{0}(x)-\lim _{x \rightarrow x_{n}^{+}} u_{0}(x)\right|>\delta\right\} .
\end{gathered}
$$

Now we define the approximated initial function $u_{0}^{\delta}$ by

$$
u_{0}^{\delta}(x)= \begin{cases}\lim _{x \rightarrow-\infty} u_{0}(x), & x<x_{1}, \\ u_{0}\left(x_{n}\right), & x \in\left[x_{n}, x_{n+1}\right\rangle, \\ \lim _{x \rightarrow \infty} u_{0}(x), & x>x_{M} .\end{cases}
$$

We have that $\left\|u_{0}^{\delta}-u_{0}\right\|_{L_{1}} \rightarrow 0$ as $\delta \rightarrow 0$. At each discontinuity in $u_{0}^{\delta}$ we construct the $\delta$-approximation to the solution of the Riemann problem defined by $\left(u_{0}\left(x_{n-1}\right), u_{0}\left(x_{n}\right)\right)$. When two $\delta$-approximations interact at some $t>0$, we are still in the class of step functions with compact support and a finite number of steps. Therefore the process can be repeated. With a slight abuse of notation we will call this "solution" $u_{\delta}$. 
It is clear that this process can be repeated an arbitrary number of times. If we define the $t_{i}$ as the $i$ th time discontinuities collide, we can continue our appproximation up to a time $T=\lim _{i \rightarrow \infty} t_{i}$. In order to remove this restriction we will make a modification of our method: We construct our solution up to some time $t_{N}$. At $t_{N}$ we will not use $\delta$-approximations to "solve" the Riemann problems, but we will use an approximation where some of the small waves originating from these are ignored. The next time discontinuities collide we will again use $\delta$-approximations until some new $t_{N^{\prime}}$, and the process is repeated. We will show that it is sufficient to do this a finite number of times depending on $\delta$ in order to carry our approximation up to infinite time. In order to show that this is a well-defined construct we need some lemmas.

We follow the notation in $[14$, p. 370]. By

$$
\left(u_{l}, u_{r}\right)=\left[\left(u_{0}, \ldots, u_{n}\right) /\left(\varepsilon_{1}, \ldots, \varepsilon_{n}\right)\right]
$$

we mean that $u_{k}$ is connected to $u_{k-1}$ by a $k$-shock or a $k$-rarefaction wave with strength $\left|\varepsilon_{k}\right|$, i.e., $u_{k}=U^{(k)}\left(u_{k-1}, \varepsilon_{k}\right)$, and $u_{l}=u_{0}$ and $u_{n}=u_{r}$. Now let $u_{l}, u_{m}, u_{r}$ be given states near a given state $u$, and let

$$
\begin{aligned}
& \left(u_{l}, u_{m}\right)=\left[\left(u_{0}, \ldots, u_{n}\right) /\left(\alpha_{1}, \ldots, \alpha_{n}\right)\right], \\
& \left(u_{m}, u_{r}\right)=\left[\left(u_{0}, \ldots, u_{n}\right) /\left(\beta_{1}, \ldots, \beta_{n}\right)\right] .
\end{aligned}
$$

With these definitions in hand we can prove the following slight modification of [14, Theorem 19.2] or [2, Theorem 2.1].

Lemma 1. Assume that a discontinuity $\alpha$ (in our scheme) of family $j$ separating $\left(u_{l}, u_{m}\right)$ and a discontinuity $\beta$ offamily $k$ separating $\left(u_{m}, u_{r}\right)$ collide and that (2.4), (2.5), and (2.6) hold. Then

$$
\varepsilon_{i}=\delta_{i j} \alpha+\delta_{i k} \beta+O(1)|\alpha||\beta| .
$$

Proof. The proof of this is the same as the proof of Theorem 2.1 in [2].

Let $t_{i}$ be the $i$ th time discontinuities in $u_{\delta}$ collide, and let $\left|\varepsilon_{i}^{j}\right|$ be the strength of the $j$ th discontinuity from the left in the strip $t_{i}<t<t_{i+1}$. We say two discontinuities in $u_{\delta}$ are approaching if the speed of the one on the left is larger than the speed of the one on the right. We define

$$
\begin{aligned}
T_{k} & =\sum_{j}\left|\varepsilon_{k}^{j}\right|, \\
Q_{k} & =\sum\left|\alpha_{k}\right|\left|\beta_{k}\right|
\end{aligned}
$$

where the sum in $Q_{k}$ is taken over all approaching pairs in $t_{k}<t<t_{k+1}$.

Lemma 2. Let $m, n>0$. If $n>m$ and $T_{m}$ is sufficiently small, then

$$
Q_{n} \leq Q_{m}, \quad T_{n}+K Q_{n} \leq T_{m}+K Q_{m}
$$

for some $K>0$.

Proof. The proof is similar in spirit to the proof of the corresponding theorem for Glimm's construction, see [2] or [14]. We first assume that $n=m+1$. Let the collision at $t_{m+1}$ take place at $x$ (if there are several collisions we can use the same argument at each collision). Since discontinuities propagate at finite speed, we can find $\varepsilon>0$ and an interval $J$ such that $x \in J$, and at $t_{m+1}-\varepsilon$ all 


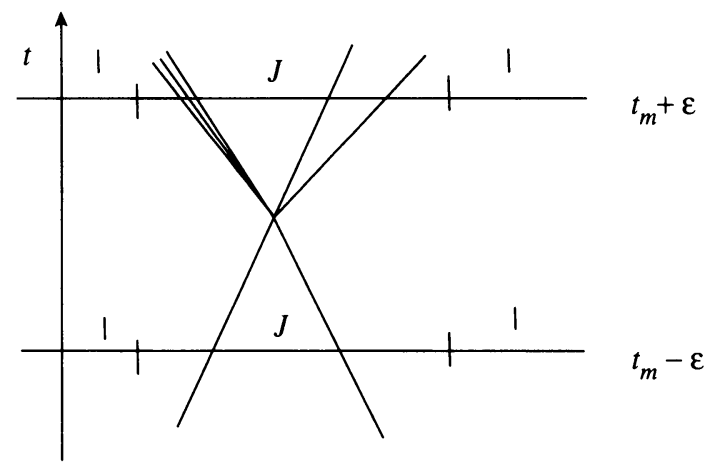

FIGURE 1

colliding discontinuities are in $J$, and at $t_{m+1}+\varepsilon$ all discontinuities emanating from $\left(x, t_{m+1}\right)$ are in $J$. These are also the only discontinuities in $J$ in the time interval $\left[t_{m+1}-\varepsilon, t_{m+1}+\varepsilon\right]$. Let $I=\mathbb{R} \backslash J$, cf. Figure 1 . Let $T_{k}(I)$ be $T_{k}$ with the summation restricted to $I$, similarly for $Q_{k}(I)$. From Lemma 1 we have

$$
T_{n} \leq T_{m}+K_{0} Q_{m}(J), \quad Q_{n}=Q_{n}(I)+Q_{n}(I, J)
$$

where $Q_{k}(I, J)$ is the sum with one wave from $I$ and the other from $J$.

$$
\begin{aligned}
Q_{n}(I, J) & =\sum_{\delta \text { appr } \varepsilon_{n}}\left|\varepsilon_{n}\right||\delta| \leq \sum_{\delta \text { appr } \alpha \text { or } \beta}(|\alpha|+|\beta|)|\delta|+K_{0} Q_{m}(J) T_{m}(I) \\
& \leq Q_{m}(I, J)+K_{0} Q_{m}(J) T_{m} \leq Q_{m}(I, J)+\frac{1}{2} Q_{m}(J) \quad \text { if } K_{0} T_{m} \leq \frac{1}{2} .
\end{aligned}
$$

Therefore

$$
\begin{aligned}
Q_{n}-Q_{m} & =\left[Q_{n}(I)+Q_{n}(I, J)\right]-\left[Q_{m}(I)+Q_{m}(J)+Q_{m}(I, J)\right] \\
& \leq Q_{m}(I, J)+\frac{1}{2} Q_{m}(J)-Q_{m}(J)-Q_{m}(I, J)=-\frac{1}{2} Q_{m}(J) \leq 0
\end{aligned}
$$

Now

$$
\begin{aligned}
T_{n}+K Q_{n} & \leq T_{m}+K_{0} Q_{m}(J)+K Q_{m}-(K / 2) Q_{m}(J) \\
& \leq T_{m}+K Q_{m} \text { if } K_{0}-K / 2 \leq 0
\end{aligned}
$$

Summing we have that the inequalities hold for any $n>m$.

Corollary 1. If T.V.( $\left(u_{0}\right)$ is sufficiently small then

$$
\operatorname{osc} u_{\delta} \leq T . V .\left(u_{\delta}\right) \leq c T_{n} \leq \bar{c} T_{0} \leq \overline{\bar{c}} T . V .\left(u_{0}\right)
$$

where all constants are independent of $t$ and $\delta$.

Proof. osc $\leq T . V$. is always true. T.V. $\leq c T$ since they are equivalent norms. $T_{n} \leq T_{n}+K Q_{n} \leq T_{0}+K Q_{0} \leq T_{0}+K T_{0}^{2} \leq 2 T_{0}$ if $K T_{0} \leq 1$

Now define

$$
P_{k}=\sum\left|\varepsilon_{k}^{j-1}\right|\left|\varepsilon_{k}^{j}\right|
$$

where the sum is taken over those discontinuities that collide at $t_{k}$ (typically $\left.P_{k}=\left|\varepsilon_{k}^{j-1}\right|\left|\varepsilon_{k}^{j}\right|\right)$. 
Lemma 3. $\sum_{i} P_{i}<\infty$.

Proof. If $\Delta Q_{k}=Q_{k}-Q_{k+1}$, then by (2.7) $\Delta Q_{k} \geq \frac{1}{2} P_{k}$. Therefore

$$
Q_{0} \geq Q_{0}-Q_{k}=\sum^{k} \Delta Q_{i} \geq \frac{1}{2} \sum^{k} P_{i}
$$

Now we can define $t_{N_{1}}$, where $N_{1}<\infty$ is the smallest number such that $P_{k}<$ $\delta$ for all $k \geq N_{1}$, and we relabel the collision times up to $t_{N_{1}}: t_{0}^{1}, t_{1}^{1}, \ldots, t_{N_{1}}^{1}$. At the collision(s) at $t_{N_{1}}^{1}$, we do not construct waves of families different from the ones that are colliding, although we allow these to change their speeds. What this means is perhaps best illustrated by an example: Assume that the collision at $t_{N_{1}}^{1}$ is between a discontinuity of family $l$ and one of family $m$. Assume also that the solution of the Riemann problem defined by the states to the left and right of this collision has a solution that contains waves of families $k$, $m, n$, and $l$. Note that Lemma 1 says that the waves of families $k$ and $n$ will be small. In making the step function approximation to the solution of the Riemann problem the small waves of family $n$ and $k$ are ignored and the solution is regarded as constant over these waves.

This constant is chosen as follows: If the small wave is of a family smaller than the familiy of both the colliding waves, i.e., its speed is strictly smaller than the speeds of the colliding waves, then the state to the right of this wave is set equal to the state to the left of it. Similarly if the family of the wave to be ignored is larger than the families of the colliding waves, then the state to the left of the small wave is set equal to the state to the right of it. If the familiy of the small wave is between the families of the colliding waves, we may choose either to set the state to the left of it equal to the state on the right, or vice versa.

This of course introduces an additional error into our approximation, but it is neccesary to remove some discontinuities in order to limit the number of fronts to track. In Lemmas 5 and 6 we show that this error is so small that the approximation remains an approximate weak solution.

The next collision time after $t_{N_{1}}^{1}$ we label $t_{0}^{2}$. At this collision we again use the original approximation technique where all waves in the solution of a Riemann problem are approximated. We continue using this approximation up to a collision time $t_{N_{2}}^{2}$, where $N_{2}$ is defined like $N_{1}$. When solving the Riemann problems at $t_{N_{2}}^{2}$ we again ignore waves of new families. Continuing in this way we get collision times for the $u_{\delta}$

$$
t_{0}^{1}, \ldots, t_{N_{1}}^{1}, t_{0}^{2}, \ldots, t_{N_{2}}^{2}, t_{0}^{3}, \ldots, t_{N_{3}}^{3}, \ldots, t_{0}^{i}, \ldots, t_{N_{i}}^{i}, \ldots
$$

Lemma 2 was shown only for collisions at $t_{j}^{i}$ where $j<N_{i}$, but it is easily seen that $Q_{n}$ and $T_{n}+K Q_{n}$ are decreasing also for the collisions at $t_{N_{i}}^{i}$. Hence Lemma 3 will hold when the sum is taken over all collisions. We restate this as

$$
\sum_{i} \sum_{k=0}^{N_{i}} P_{k}^{i}<\infty
$$

where $P_{k}^{i}$ refers to the collision(s) taking place at $t_{k}^{i}$. Therefore there is an integer $M$ such that $\sum_{k=0}^{N_{i}} P_{k}^{i} \leq \delta$ for all $i \geq M$. For such $i$ we have that 
$P_{k}^{i} \leq \delta$ for all $k$. Hence, after $t_{N_{M-1}-1}^{M-1}$ we do not create waves of new families at collision points. Since we have a strictly hyperbolic system where the speeds of waves of different families are different, we see that after a certain time all discontinuities will have passed through each other and there will be no more interactions. Thus the approximation $u_{\delta}$ can be defined at any $(x, t)$ in the upper halfplane.

We remark that the above reasoning could also be used on the following approximation strategy: If $P_{k}$ is less than $\delta$ for some $k$, we do not construct waves of new families at $t_{k}$, and we define $t_{0}^{2}$ to be the first time after $t_{k}$ that discontinuities existing already before $t_{k}$ collide. This strategy may be more practical and one can also show (as we will do) that it gives an approximate weak solution.

\section{RESULTS}

Corollary 2. If T.V. $\left(u_{0}\right)$ is small then

$$
T . V \cdot x\left(u_{\delta}\right)+\sup _{x}\left|u_{\delta}\right| \leq c T . V \cdot\left(u_{0}\right)
$$

where $c$ is independent of $t$ and $\delta$.

This corollary is a consequence of Corollary 1 , and its proof may be found in $[14$, p. 384$]$.

\section{Corollary 3.}

$$
\left\|u_{\delta}\left(\cdot, t_{1}\right)-u_{\delta}\left(\cdot, t_{2}\right)\right\|_{L_{1}} \leq c\left|t_{2}-t_{1}\right|
$$

where $c$ is independent of $\delta, t_{1}$, and $t_{2}$.

Proof. Let $M<\infty$ be the maximum speed at which a wave may propagate. Thus, if $t_{1}<t_{2}$ then $\left|u_{\delta}\left(x, t_{2}\right)-u_{\delta}\left(x, t_{1}\right)\right|$ is bounded by the spatial variation of $u_{\delta}\left(y, t_{1}\right)$ over the interval $\left(x-M\left|t_{2}-t_{1}\right|\right)<y<\left(x+M\left|t_{2}-t_{1}\right|\right)$. However, $u_{\delta}(\cdot, t)$ is of bounded variation, so that we may write

$$
\int_{-\infty}^{\infty}\left|u_{\delta}\left(x, t_{2}\right)-u_{\delta}\left(x, t_{1}\right)\right| d x=O(1) \int_{-\infty}^{\infty} \int_{x-M\left|t_{2}-t_{1}\right|}^{x+M\left|t_{2}-t_{1}\right|}\left|\frac{d u_{\delta}}{d y}\right| d x d y .
$$

Here, $\left|d u_{\delta} / d y\right| d x d y$ is a measure of mass $T \cdot V \cdot u_{\delta}(x, t)$, and by changing the order of integration we have

$$
\begin{aligned}
\int_{-\infty}^{\infty}\left|u_{\delta}\left(x, t_{2}\right)-u_{\delta}\left(x, t_{1}\right)\right| d x & =O(1) M\left|t_{2}-t_{1}\right| T . V \cdot u_{\delta}(x, t) \\
& \leq O(1) M\left|t_{2}-t_{1}\right| T . V . u_{0, \delta}(x),
\end{aligned}
$$

where the last inequality holds by Corollary 1 .

Now we have that the $u_{\delta}$ functions satisfy

$$
\begin{aligned}
\left\|u_{\delta}(\cdot, \cdot)\right\|_{\infty} & \leq M_{1}, \\
T \cdot V_{\cdot x}\left(u_{\delta}(\cdot, t)\right) & \leq M_{2}, \\
\left\|u_{\delta}\left(\cdot, t_{1}\right)-u_{\delta}\left(\cdot, t_{2}\right)\right\|_{L_{1}} & \leq M_{3}\left|t_{2}-t_{1}\right| .
\end{aligned}
$$

The constants $M_{i}$ are independent of the $\delta$ and the times $t_{1}$ and $t_{2}$. Using Helly's theorem as in [14] one can show that (3.1) to (3.3) imply the following 
Theorem 3. If (3.1)-(3.3) hold then a subsequence of the family $\left\{u_{\delta}\right\}$ converges in $L_{1}^{\text {loc }}$. For this subsequence $f\left(u_{\delta}\right) \rightarrow f(u)$ in $L_{1}^{\text {loc }}$, where $u$ is the limit function.

We use this theorem for the sequence of functions $\left\{u_{\delta}\right\}$ as $\delta \rightarrow 0$. Now we have to check whether the limit is a weak solution to our problem. To this end we define

$$
\mathscr{I}_{\phi}(u, f)=\int_{0}^{\infty} \int_{-\infty}^{\infty}\left(\phi_{t} u+\phi_{x} f(u)\right) d x d t+\int_{-\infty}^{\infty} \phi(x, 0) u_{0}(x) d x
$$

and

$$
\begin{aligned}
\mathscr{F}_{\phi}^{t_{1}, t_{2}}(u, f)= & \int_{t_{1}}^{t_{2}} \int_{-\infty}^{\infty}\left(\phi_{t} u+\phi_{x} f(u)\right) d x d t \\
& +\int_{-\infty}^{\infty} \phi\left(x, t_{1}\right) u\left(x, t_{1}\right) d x-\int_{-\infty}^{\infty} \phi\left(x, t_{2}\right) u\left(x, t_{2}\right) d x
\end{aligned}
$$

for all smooth $\phi$ with compact support in $\mathbb{R} \times \mathbb{R}_{0}^{+}$. We now fix $\delta$ and $\phi$. Let $s, t$ be consecutive times when discontinuites of $u_{\delta}$ collide. If we had tracked all waves from the Riemann problems in $u_{\delta}(x, s)$, we could compare it with an exact solution in the strip $[s, t]$, since the exact solution here would be a series of noninteracting solutions to Riemann problems. Comparing $u_{\delta}$ with this exact solution we would get an "error" estimate, telling us how far $u_{\delta}$ is from being a weak solution. But we cannot do this directly, since we do not neccesarily know the weak solution in the whole strip $\langle t, s\rangle$. Therefore we define $v_{\delta}$ to be a $\delta$-approximation to the initial value problem

$$
v_{t}+f(v)_{x}=0, \quad v(x, s)=u_{\delta}(x, s) .
$$

Let $t_{0}=s$. Since discontinuities of $v_{\delta}$ propagate with finite speed, they will either collide at some $t_{1}<t$, or else not collide until $t$, in which case we define $t_{1}=t$. If $t_{1}<t$ then from $t_{1}$ we define $v_{\delta}$ to be the $\delta$-approximation to the same problem with initial values $u_{\delta}\left(x, t_{1}\right)$. Now either the discontinuites of $v_{\delta}$ will either not collide until $t$, in which case we set $t_{2}=t$, or else collide at some $t_{2}<t$. We can continue in this fashion to obtain a sequence $\left\{t_{i}\right\}$. We have that either this sequence is finite and $t_{k}=t$ for some $k$, or else $\lim _{i} t_{i}=t$. To see this, let $d_{i}$ denote the smallest distance between discontinuities of $u_{\delta}\left(\cdot, t_{i}\right)$. Note that $d_{i}>0$ if $t_{i} \neq t$. But since discontinuities of $v_{\delta}$ have finite speed we have that $d_{i} \leq M\left(t_{i+1}-t_{i}\right)$, where $M$ is a bound on the speed of discontinuities. Thus we see that $\lim t_{i} \geq t$. Thus we have filled the interval $[t, s]$ with at most countably many intervals $\left[t_{i}, t_{i+1}\right]$ such that $t-s=\sum\left(t_{i+1}-t_{i}\right)$, cf. Figure 2 .

In the strip $\left[t_{i}, t_{i+1}\right]$ we define $v(x, t)$ to be the weak solution of

$$
\begin{gathered}
v_{t}+f(v)_{x}=0, \\
v\left(x, t_{i}\right)=v_{\delta}\left(x, t_{i}\right)=u_{\delta}\left(x, t_{i}\right) .
\end{gathered}
$$

The function $v(x, t)$ can be defined in this strip since the different Riemann problems only will interact at $t_{i+1}$. With these definitions in mind we can state the following

Lemma 4. For $t \in\left[t_{i}, t_{i+1}\right]$ we have

$$
\int_{x_{1}}^{x_{2}}\left|v-u_{\delta}\right| d x=O(\delta)\left(t-t_{i}\right) .
$$




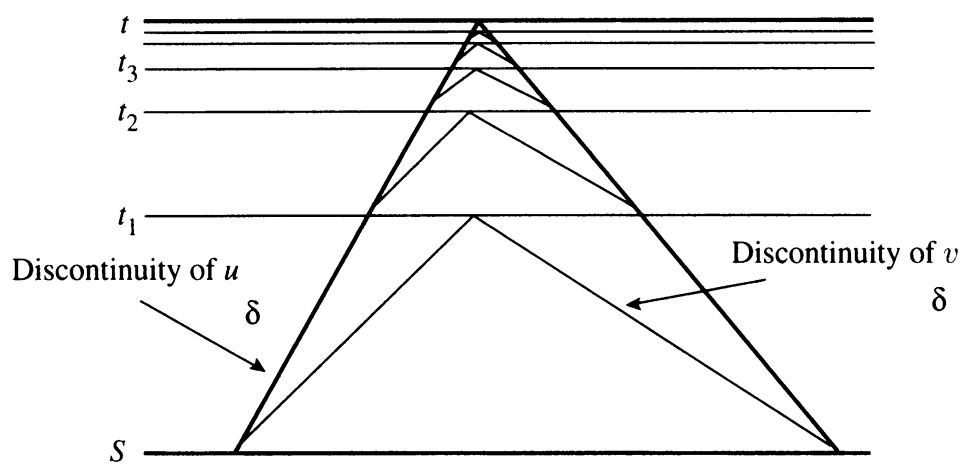

FIGURE 2

Proof.

$$
\int_{x_{1}}^{x_{2}}\left|v-u_{\delta}\right| d x \leq \int_{x_{1}}^{x_{2}}\left|v-v_{\delta}\right| d x+\int_{x_{1}}^{x_{2}}\left|v_{\delta}-u_{\delta}\right| d x
$$

For the first term note that $v=v_{\delta}$ exept when $(x, t)$ is in a rarefaction fan. In a rarefaction fan the difference $\left|v_{\delta}-v\right|$ is always less than or equal to $O(\delta)$. If $\hat{u}_{r}, \hat{u}_{l}$ are the states to the right and left of such a fan respectively, then the integral across the fan will be a sum of integrals across each step of $v_{\delta}$. The number of such steps is $O\left(\left|\hat{u}_{r}-\hat{u}_{l}\right| / \delta\right)$, and the width of each region where $v_{\delta}$ differs from $v$ is $\left(t-t_{i}\right) O(\Delta \lambda)$, where $\Delta \lambda=\left|\lambda\left(v_{j}^{(k)}\right)-\lambda\left(v_{j+1}^{(k)}\right)\right|=O(\delta)$. Therefore the first term in (3.8) is a sum over all rarefaction fans of $v_{\delta}$

$$
\sum_{j} O\left(\frac{\left|\hat{u}_{r_{j}}-\hat{u}_{l_{j}}\right|}{\delta}\right) O(\delta)\left(t-t_{i}\right) O(\delta)
$$

But this is less than

$$
T . V .\left(u_{\delta}\left(x, t_{i}\right)\right)\left(t-t_{i}\right) O(\delta) .
$$

We have that $v_{\delta}$ and $u_{\delta}$ both are step functions with a finite number of steps. Furthermore they are equal except possibly in a fan emanating from each discontinuity in $v_{\delta}\left(x, t_{i}\right)$. Let $x_{j}$ be in the $j$ th interval from the left where $v_{\delta}(x, t)$ differs from $u_{\delta}(x, t)$. We label the discontinuities not tracked in $u_{\delta}$, but tracked in $v_{\delta}$, by $\left\{\eta_{j}\right\}$, where $\left|\eta_{j}\right|$ is the strength of this discontinuity.

$$
\int_{x_{1}}^{x_{2}}\left|v_{\delta}(x, t)-u_{\delta}(x, t)\right| d x \leq \sum_{j} M\left(t-t_{i}\right)\left|v_{\delta}\left(x_{j}, t\right)-u_{\delta}\left(x_{j}, t\right)\right|
$$

(here $M$ is a constant such that $M>\sup _{u \in C}\left|\lambda_{n}(u)-\lambda_{1}(u)\right|, C=$ convex hull of $\left.\left\{\operatorname{Ran}\left\{u_{\delta}\right\}\right\}\right)$

$$
\begin{aligned}
& =M\left(t-t_{i}\right) \sum_{j}\left|v_{\delta}\left(x_{j}, t\right)-u_{\delta}\left(x_{j}, t\right)\right| \\
& =M\left(t-t_{i}\right) O\left(\sum_{j}\left|\eta_{j}\right|\right)
\end{aligned}
$$

But by construction of $u_{\delta}$ we have that $\sum_{j}\left|\eta_{j}\right| \leq O(\delta)$ and the result follows. 
Lemma 5. If we let $t_{i}, t_{i+1}$ be as before we have

$$
\mathscr{J}_{\phi}^{t_{i}, t_{i+1}}\left(u_{\delta}, f\right)=O(\delta)\left(\left(t_{i+1}-t_{i}\right)+\left(t_{i+1}-t_{i}\right)^{2}\right) .
$$

Proof. Let $M \geq \sup \left\{\left|\phi_{x}\right|,\left|\phi_{t}\right|,|\phi|,|d f|\right\}$, and let $v(x, t)$ be as before; then

$$
\begin{aligned}
& \left|\mathcal{F}_{\phi}^{t_{i}, t_{i+1}}\left(u_{\delta}, f\right)\right|=\left|\mathcal{J}_{\phi}^{t_{i}, t_{i+1}}\left(u_{\delta}, f\right)-\mathscr{F}_{\phi}^{t_{i}, t_{i+1}}(v, f)\right| \\
& \quad=\mid \int_{t_{i}}^{t_{i+1}} \int\left(\left(u_{\delta}-v\right) \phi_{t}+\left(f\left(u_{\delta}\right)-f(v)\right) \phi_{x} d x d t-\int \phi\left(x, t_{i+1}\right)\left(u_{\delta}-v\right) d x \mid\right. \\
& \quad \leq M\left(\int_{t_{i}}^{t_{i+1}} \int\left|u_{\delta}-v\right| d x d t+\int\left|u_{\delta}-v\right| d x+\int_{t_{i}}^{t_{i+1}} \int\left|f\left(u_{\delta}\right)-f(v)\right| d x d t\right) .
\end{aligned}
$$

Now

$$
f\left(u_{\delta}\right)-f(v)=d f\left(u_{\delta}-v\right)+O^{2}\left(u_{\delta}-v\right)
$$

and

$$
\int\left|f\left(u_{\delta}\right)-f(v)\right| d x \leq M \int\left|u_{\delta}-v\right| d x+\int\left|O^{2}\left(u_{\delta}-v\right)\right| d x
$$

Using (3.10) in (3.9), Lemma 4 on (3.9), and integrating in $t$ will give Lemma 5.

Lemma 6. If $t$ and $s$ are as before we have

$$
\mathscr{J}_{\phi}^{t, s}\left(u_{\delta}, f\right) \leq O(\delta)\left((s-t)+(s-t)^{2}\right) .
$$

Proof. By Lemma 5

$$
\mathscr{I}_{\phi}^{t, s}\left(u_{\delta}, f\right)=O(\delta) \sum\left(\left(t_{i+1}-t_{i}\right)+\left(t_{i+1}-t_{i}\right)^{2}\right)
$$

where $t_{i}$ is as before. We have $\sum\left(t_{i+1}-t_{i}\right)=(s-t)$, which means that the second term in the sum is less than $(s-t)^{2}$.

Lemma 7. $\lim _{\delta \rightarrow 0} \mathscr{J}_{\phi}\left(u_{\delta}, f\right)=0$.

Proof. If we let $s_{i}, s_{i+1}$ be consecutive times when discontinuities of $u_{\delta}$ collide we have

$$
\mathscr{I}_{\phi}\left(u_{\delta}, f\right)=\sum_{i} \mathscr{J}_{\phi}^{s_{i}, s_{i+1}}\left(u_{\delta}, f\right)
$$

therefore by Lemma 6

$$
\mathscr{I}_{\phi}\left(u_{\delta}, f\right)=O(\delta) \sum\left(\left(s_{i+1}-s_{i}\right)+\left(s_{i+1}-s_{i}\right)^{2}\right) .
$$

We now have $\sum\left(s_{i+1}-s_{i}\right) \leq T$ where $T$ is such that supp $\phi$ is contained in $\{t \leq T\}$. Therefore the sum in (3.11) is finite, and the lemma follows.

Thus $u_{\delta}$ converges to a weak solution, and we have proved the following theorem. 
Theorem 4. Assume $f: \mathbb{R}^{n} \rightarrow \mathbb{R}^{n}$ is strictly hyperbolic and genuinely nonlinear and $u_{0}: \mathbb{R} \rightarrow \mathbb{R}^{n}$ is such that T.V.x $\left(u_{0}\right)$ is sufficiently small. Then there exists a weak solution $u(x, t)$ to the initial value problem

$$
u_{t}+f(u)_{x}=0, \quad u(x, 0)=u_{0}(x) .
$$

For the solution of the Riemann problem we have that all discontinuities satisfy the Lax entropy conditions:

$$
\lambda_{k}\left(u_{r}\right)<s_{k} \leq \lambda_{k+1}\left(u_{r}\right), \quad \lambda_{k-1}\left(u_{l}\right) \leq s_{k}<\lambda_{k}\left(u_{l}\right)
$$

where $s_{k}$ is the speed of a $k$-shock. Since discontinuities of $u_{\delta}$, at least if they have a magnitude larger than $\delta$, almost (in the sense of (3.14) and (3.15)) satisfy these conditions, one may ask whether discontinuities in the limit function satisfy (3.13). The next theorem gives a partial answer to this. If $u(x, t)$ has a discontinuity at $(x, t)$, and there is a neighbourhood $N$ of $(x, t)$ such that we can find a smooth curve $x(s)$ in $N$ where $x(t)=x$, and in $N$ we can define

$$
u_{+}(t)=\lim _{x \rightarrow x(t)^{+}} u(x, t), \quad u_{-}(t)=\lim _{x \rightarrow x(t)^{-}} u(x, t),
$$

and $u_{ \pm}$are continuous in $N$, and $u_{-}(t) \neq u_{+}(t)$ for $t$ in $N$, then we say that the discontinuity at $(x, t)$ is isolated.

Theorem 5. An isolated discontinuity in $u=\lim _{\delta \rightarrow 0} u_{\delta}$ moving with a speed $s(t)$ satisfies (3.13) for some $k$.

Proof. Assume that for a fixed time $t$ we have an isolated discontinuity in the limit function $u$ at $x$ moving with a speed $s$. Let

$$
u_{l}=\lim _{x \rightarrow x^{-}} u(x, t), \quad u_{r}=\lim _{x \rightarrow x^{+}} u(x, t) .
$$

Since convergence in $L_{1}^{\text {loc }}$ implies pointwise convergence almost everywhere, we can find sequences $\left\{x_{\delta, l, 1}\right\},\left\{x_{\delta, r, 1}\right\},\left\{x_{\delta, l, 2}\right\},\left\{x_{\delta, r, 2}\right\}$, and $\left\{t_{\delta, 1}\right\}$ and $\left\{t_{\delta, 2}\right\}$ with $t_{\delta, 1}<t_{\delta, 2}$ such that

$$
\begin{aligned}
& t_{\delta}, k \rightarrow t, \quad x_{\delta, l, k} \rightarrow x^{-}, \quad x_{\delta, r, k} \rightarrow x^{+}, \\
& u_{\delta}\left(x_{\delta, l, k}, t_{\delta, k}\right) \rightarrow u_{l}, \quad u_{\delta}\left(x_{\delta, r, k}, t_{\delta, k}\right) \rightarrow u_{r}
\end{aligned}
$$

as $\delta \rightarrow 0$ for $k=1,2$. Furthermore we may define a paralellepiped $E_{\delta}$ with corners $\left(x_{\delta, l, 1}, t_{\delta}^{1}\right),\left(x_{\delta, r, 1}, t_{\delta}^{1}\right),\left(x_{\delta, r, 2}, t_{\delta}^{2}\right)$, and $\left(x_{\delta, l, 2}, t_{\delta}^{2}\right)$ such that the diagonals have slope not too different from $s$, i.e.,

$$
\frac{x_{\delta, r, 1}-x_{\delta, l, 2}}{t_{\delta}^{1}-t_{\delta}^{2}}-\varepsilon(\delta)<s<\frac{x_{\delta, l, 1}-x_{\delta, r, 2}}{t_{\delta}^{1}-t_{\delta}^{2}}+\varepsilon(\delta),
$$

for some $\varepsilon(\delta)$ that vanishes as $\delta \rightarrow 0$.

We call a discontinuity line in $u_{\delta}$ an approximate shock wave if it represents a shock in the solution to the Riemann problem where it originated. Similarly we call a discontinuity line an approximate rarefaction wave if it represents part of a rarefaction fan. These are the only kind of discontinuity lines in $u_{\delta}$. Let

$$
M_{\delta}^{k}=\frac{\sum_{i}\left|\eta_{i}\right|}{x_{\delta, r, k}-x_{\delta, l, k}}
$$

where the sum is taken over the approximate rarefaction waves crossing the line segment $t_{\delta}^{k} \times\left[x_{\delta, l, k}, x_{\delta, r, k}\right]$ for $k=1,2$. If this is unbounded as $\delta \rightarrow 0$ then 
the limit function $u$ must have a rarefaction wave centered at $(x, t)$ and the discontinuity would not be isolated here. Thus $M_{\delta}^{k}$ is bounded as $\delta \rightarrow 0$. Now

$$
\begin{aligned}
\frac{\left|u_{\delta, r, k}-u_{\delta, l, k}\right|}{x_{\delta, r, k}-x_{\delta, l, k}} & \leq \frac{\sum \mid \text { appr. rarefaction }\left|+\sum\right| \text { appr. shock } \mid}{x_{\delta, r, k}-x_{\delta, l, k}} \\
& =M_{\delta}^{k}+\frac{\sum \mid \text { appr. shock } \mid}{x_{\delta, r, k}-x_{\delta, l, k}} .
\end{aligned}
$$

Since the fraction on the left is unbounded as $\delta \rightarrow 0$, there must be approximate shocks crossing the line segment $t_{\delta}^{k} \times\left[x_{\delta, l, k}, x_{\delta, r, k}\right]$ for such small $\delta$.

Since the discontinuity is isolated, it follows that the total strength of all approximate waves of $u_{\delta}$ crossing the left and right sides of $E_{\delta}$ must vanish as $\delta \rightarrow 0$.

We now define a shock line to be a sequence of approximate shock waves of the same family in $u_{\delta}$. Assuming that a shock line has been defined for $t<t_{n}$, where $\left\{t_{n}\right\}$ are the collision times of $u_{\delta}$, and in the strip $t_{n}<t<t_{n+1}$ consists of the approximate shock $\eta$. If $\eta$ does not collide at $t_{n+1}$ then the shock line continues as $\eta$ until $t_{n+2}$. If $\eta$ collides at $t_{n+1}$ the shock line stops if the approximate solution of the Riemann problem defined by the collision of $\eta$ does not contain an approximate shock wave of the same family as $\eta$. If the approximated solution of this Riemann problem contains an approximate shock wave of the same family as $\eta$, it continues as that approximate shock wave. Note that to each shock line there corresponds one family.

It now follows that there must be shock lines in $E_{\delta}$ that do not intersect the left or right side of $E_{\delta}$ and that the state to the left of the leftmost of these must tend to $u_{l}$ as $\delta$ tends to zero. To see this, label this state $v_{l}$, and let the position of this approximated shock line at $t_{\delta}^{k}$ be $y_{l}$, then $\left|u_{l}-v_{l}\right| \leq$ $\left|u_{l}-u_{\delta, l, k}\right|+\left|v_{l}-u_{\delta, l, k}\right|$. The first of these two terms tends to zero. Between $y_{l}$ and $x_{\delta, l, k}, u_{\delta}$ only varies over discontinuities that are either approximated rarefaction waves and therefore arbitrarily small as $\delta$ becomes small, or are shock lines that leave $E_{\delta}$ through the left or right side, and the strength of these must also be arbitrarily small as $\delta$ tends to zero. Similarly the state to the right of these shock lines must tend to $u_{r}$ as $\delta$ tends to zero.

Since $\varepsilon(\delta)$ vanishes as $\delta \rightarrow 0$, the family of all shock lines not intersecting the left or right sides of $E_{\delta}$ must be the same, say $k$, since our system is strictly hyperbolic. Furthermore the speed of the approximated shock waves which constitute these shock lines must tend to $s$ as $\delta \rightarrow 0$. Any approximate shock wave of family $k$ and left and right states $v_{l}$ and $v_{r}$ respectively, will satisfy the approximate Lax inequalities

$$
\begin{aligned}
& \lambda_{k-1}\left(v_{l}\right)<\tilde{s}+O(\delta)<\lambda_{k}\left(v_{l}\right), \\
& \lambda_{k}\left(v_{r}\right)<\tilde{s}+O(\delta)<\lambda_{k+1}\left(v_{r}\right),
\end{aligned}
$$

where $\tilde{s}$ is the speed of the approximate shock wave. Now the result follows by applying (3.14) to the leftmost of the shock lines contained in $E_{\delta}$ and (3.15) to the rightmost.

After a certain time, we have that our approximation $u_{\delta}$ will consist of constant states with discontinuities that are moving apart. This has some similarity to the solution of a Riemann problem, and we will show that the limit function 
also has such a property. We now let $T_{\delta}$ be the last time discontinuities in $u_{\delta}$ collide. After $T_{\delta}, u_{\delta}$ will consist of a number of states; $\left\{u_{i}\right\}_{1}^{M}$ separated by discontinuities moving apart. We define the real states of $u_{\delta}$ to be those $u_{i}$ such that $u_{i-1}$ is connected to $u_{i}$ by a different wave than the one connecting $u_{i}$ to $u_{i+1}$. We label the real states of $u_{\delta}:\left\{\bar{u}_{i}\right\}_{1}^{N} \subset\left\{u_{i}\right\}_{1}^{M}$. Concerning the real states of $u_{\delta}$ we have the following result.

Theorem 6. For sufficiently small $\delta$, let $\left\{\bar{u}_{i}\right\}_{1}^{N}$ be the real states of $u_{\delta}$ after $T_{\delta}$. Then $N \leq n+1$ (where $n$ is the dimension of the system) and there exists some $u_{L}, u_{R}$ such that

$$
\left(u_{L}, u_{R}\right)=\left[\left(u_{0}, \ldots, u_{N^{\prime}}\right) /\left(\varepsilon_{1}, \ldots, \varepsilon_{N^{\prime}}\right)\right], \quad N \leq N^{\prime} \leq n+1,
$$

where $\left|\bar{u}_{i}-u_{i}\right| \leq O(\delta)$ for $i \leq N$.

If $\left(u_{i}, u_{i+1}\right)$ is a shock with speed $s_{i}$, then $\left(\bar{u}_{i}, \bar{u}_{i+1}\right)$ is a single discontinuity moving with speed $\bar{s}_{i}$ and $\left|\bar{s}_{i}-s_{i}\right| \leq O(\delta)$.

If $\left(u_{i}, u_{i+1}\right)$ is a rarefaction wave, which we call $u(s)$ for $s=x / t$, then $\left(\bar{u}_{i}, \bar{u}_{i+1}\right)$ is a series of approximate rarefaction waves $\left\{\left(\tilde{u}_{i_{j}}, \tilde{u}_{i_{j+1}}\right)\right\}_{j=1}^{k}$. If $\left(\tilde{u}_{i_{j}}, \tilde{u}_{i_{j+1}}\right)$ is separated by a discontinuity moving with speed $s_{i_{j}}$ then

$$
\left|u\left(s_{i_{j}}\right)-\tilde{u}_{i_{j}}\right| \leq O(\delta) \quad \text { and } \quad\left|u\left(s_{i_{j}}\right)-\tilde{u}_{i_{j+1}}\right| \leq O(\delta) .
$$

Proof. We first show that $N \leq n+1$. By construction of $u_{\delta}$ we have that the solution to the Riemann problem $\left(\bar{u}_{i}, \bar{u}_{i+1}\right)$ consists of at most $n+1$ states $\left\{v_{i}\right\}$ and that there exists a $k$ such that for $j \leq k,\left|\bar{u}_{i}-v_{j}\right| \leq O(\delta)$, and for $j>k,\left|\bar{u}_{i+1}-v_{j}\right| \leq O(\delta)$. That is, all waves in the Riemann solution are small exept the $k$-wave. Since we have no collisions in $u_{\delta}$ after $T_{\delta}$, this wave will be either a single discontinuity or a single approximate rarefaction wave by genuine nonlinearity, and because (3.14) and (3.15) will hold for an approximate shock wave. The approximation $u_{\delta}$ is constructed so that the speed of this $k$-wave will be close to the speed of the approximate wave; for a shock the speeds will be the same, for a rarefaction the speed of the head or tail of the wave may differ from the correct speed by at most $O(\delta)$. For sufficiently small $\delta$ we have that $N \leq n+1$ since $\bar{s}_{i}<\bar{s}_{i+1}$.

To prove the second statement we use induction on $N$; the number of real states in $u_{\delta}$ after $T_{\delta}$. In case $N=2$ we have just seen that the theorem holds. Assume it to be true for $N$. By "near" we will in the following mean $O(\delta)$. By construction of $u_{\delta}$ and what was said in the last paragraph we have states $\left\{v_{l}, v_{r}\right\}$ such that $\left(v_{l}, v_{r}\right)$ is near $\left(\bar{u}_{N}, \bar{u}_{N+1}\right)$, and that the Riemann problem $\left(v_{l}, v_{r}\right)$ is solved by a $j$-wave, where $j>N-1$. By the induction hypothesis, we also have states $\left(u_{1}, \ldots, u_{N}\right)$ near $\left(\bar{u}_{1}, \ldots, \bar{u}_{N}\right)$ such that the Riemann problem $\left(u_{1}, u_{N}\right)$ is solved by $\left(u_{1}, \ldots, u_{N}\right)$. We now have states $u_{N}, v_{l}$, and $v_{r}$, such that $u_{N}$ is near $v_{l}$, and $v_{l}$ is connected to $v_{r}$ by a $j$-wave. Assume that we can find a state $u_{N+1}$ near $v_{r}$, and that $u_{N}$ is connected to $u_{N+1}$ by a $j$-wave. Then

$$
\left|\bar{u}_{N+1}-u_{N+1}\right| \leq\left|\bar{u}_{N+1}-v_{r}\right|+\left|v_{r}-u_{N+1}\right| \leq 2 O(\delta),
$$

and the induction step will be completed.

To show the existence of such a state we consider two cases: First we assume that the $j$-wave is a rarefaction wave. In this case $v_{r}$ lies on an orbit starting from $v_{l}$ of the vector field of the right eigenvector $r_{j}$. Orbits starting from nearby points will stay close to the orbit starting from $v_{l}$ for small rarefaction 
strengths. Such a state can therefore be found if the wave is sufficiently weak. This will be the case if $T . V .\left(u_{0}\right)$ is sufficiently small.

If the $j$-wave is a shock, then $v_{l}, v_{r}$ will satisfy the Hugoniot relation

$$
s_{j}\left(v_{r}-v_{l}\right)=f\left(v_{r}\right)-f\left(v_{l}\right) .
$$

By continuity of $f$, we can find a state $u_{N+1}$ near $v_{r}$, and a speed $\tilde{s}_{N}$ near $s_{j}$, such that (3.16) holds for the triplet $\left\{u_{N}, u_{N+1}, \tilde{s}_{N}\right\}$. By the continuity of the eigenvalues of $d f$ this triplet will also satisfy (3.13). The proof of the statement for rarefaction waves is similar.

There is an immediate corollary of this:

Corollary 4. Let $u=\lim _{\delta \rightarrow 0} u_{\delta}, \lim _{x \rightarrow-\infty} u_{0}(x)=u_{L}$, and $\lim _{x \rightarrow \infty} u_{0}(x)=$ $u_{R}$. As $t \rightarrow \infty, u$ will consist of a finite number of constant states $\left\{u_{i}\right\}_{1}^{N}$, separated by rarefaction waves or shocks, where $N \leq n+1$. These states are the states in the solution to the Riemann problem $\left(u_{L}, u_{R}\right)$, and they will be separated by the same waves as the corresponding states in the solution to the Riemann problem.

Proof. By the proof of the preceding theorem, we can define a function $\bar{u}_{\delta}(x, t)$ for $t>T_{\delta}$ such that $\bar{u}_{\delta}$ consists of $N$ constant states separated by shocks or rarefaction waves. Let $\|\cdot\|_{1}$ denote the $L_{1}^{\text {loc }}(\mathbb{R})$ norm. As $\delta \rightarrow 0$ the difference $\left\|u_{\delta}-\bar{u}_{\delta}\right\|_{1} \rightarrow 0$. Therefore

$$
\left\|u-\bar{u}_{\delta}\right\|_{1} \leq\left\|u-u_{\delta}\right\|_{1}+\left\|u_{\delta}-\bar{u}_{\delta}\right\|_{1} \text {. }
$$

Here both terms on the right tend to zero as $\delta \rightarrow 0$. Note that $u$ does not necessarily become equal to some $\bar{u}_{\delta}$ in finite time.

We remark that the equivalent of Corollary 4 was shown to hold for the Glimm construction by Liu in [15].

\section{ACKNOWLEDGMENT}

The author is grateful to the late Raphael Høegh-Krohn for suggesting this approach to conservation laws, to Helge Holden for careful reading of this manuscript, and to Lars Holden, Aslak Tveito, and Ragnar Winther for inspiring discussions. Thanks are also due to the referee for many useful suggestions and comments on this work.

\section{REFERENCES}

1. P. D. Lax, Hyperbolic systems of conservation laws. II, Comm. Pure Appl. Math. 10 (1957), 537-566.

2. J. Glimm, Solutions in the large for nonlinear hyperbolic systems of equations, Comm. Pure Appl. Math. 18 (1965), 697-715.

3. T. P. Liu, The deterministic version of the Glimm scheme, Comm. Math. Phys. 57 (1977), 135-148.

4. A. J. Chorin, Random choice solution of hyperbolic systems, J. Comp. Phys. 22 (1976), 517-533.

5. P. D. Lax, Shock waves and entropy, Contributions to Nonlinear Functional Analysis (E. H. Zarantonello, ed.), Academic Press, New York and London, 1971, pp. 603-635.

6. J. H. Bick and G. F. Newell, A continuum model for two-directional traffic flow, Quart. J. Appl. Math. 18 (1961), 191-204. 
7. A. J. Chorin and J. E. Marsden, A mathematical introduction to fluid dynamics, Springer, New York, 1979.

8. D. W. Peaceman, Fundamentals of numerical reservoir simulation, Elsevier, Amsterdam, 1977.

9. C. M. Dafermos, Polygonal approximation of solution to the initial value problem for a conservation law, J. Math. Anal. Appl. 38 (1972), 33-41.

10. R. J. LeVeque, A large time step shock-capturing technique for scalar conservation laws, SIAM J. Numer. Anal. 19 (1982), 1051-1073.

11. H. Holden, L. Holden, and R. Høegh-Krohn, A numerical method for first order nonlinear scalar conservation laws in one dimension, Comput. Math. Appl. 15 (1988), 595-602.

12. R. Høegh-Krohn and N. H. Risebro, The Riemann problem for a single conservation law in two space dimensions, Oslo Univ. Preprint series, 1988.

13. B. K. Swartz and B. Wendroff, Aztec: A front tracking code based on Godunovs method, Appl. Numer. Math. 2 (1986), 385-397.

14. J. Smoller, Shock waves and reaction-diffusion equations, Springer, New York, 1983.

15. T. P. Liu, Large time behavior of solutions of initial and initial-boundary problems of a general system of hyperbolic conservation laws, Comm. Math. Phys. 55 (1977), 163-177.

Department of Mathematics, University of Oslo, P.O. Box 1053, Blindern, N-0316 OsLo 3, NoRway

E-mail address: nilshr@ikaros.uio.no 